\title{
A Comprehensive Review on the Phytoconstituents from Six Species of the Genus Amaranthus
}

\author{
Mohammad Musarraf Hussain \\ Department of Pharmacy, Faculty of Life and Earth Sciences, Jagannath University, Dhaka-1100, Bangladesh
}

(Received: September 15, 2018; Accepted: January 6, 2019; Published: January 17, 2019)

\begin{abstract}
The objective of this review is to consider the phytoconstituents from six species under the genus Amaranthus (A. retroflexus, A. spinosus, A. viridis, A. caudatus, A. hypocondriacus and A. tricolor). A total of sixty five (1-65) phytoconstituents with chemical structures have been reported in this study. $A$. retroflexus consists of high number of reported phytoconstituents.
\end{abstract}

Key words: Amaranthus, Sesquiterpenes, Glucosides, Prenylpropanoids, Phenolics, Triterpnoids, Saponins, Fatty acids, Flavonoids.

\section{Introduction}

Environment is a major source of medicinal plants and these plants are using as conformist moieties for the treatment of a variety of diseases from numerous years (Hussain 2018, Hussain et al. 2016a, 2016b).These medicinal plants are carrying out curative characteristics (anti-inflammatory, anticancer, anti-microbial, anti-oxidant, and antiplasmodial action) and used as traditional medicine for the treatment of various diseases (Hussain et al., 2011, 2010, 2008; Billa et al., 2013, Ismail et al., 2010). Amaranthus is a combination genus of perennial plant (annual and short lived). A small number of Amaranthus species have been cultivated as ornamental plants, pseudocereals, and leafy vegetables. A total 60 species are belonging under this genus. Amaranthus retroflexus (Family: Amaranthaceae) is an erect and annual flowering herb native to tropical continents with height in $3 \mathrm{~m}$ and leaves $15 \mathrm{~cm}$. A. spinosus (Spiny amaranth, Family: Amaranthaceae) is a plant used for jaundice and diuretic as traditional medicine. A. viridis (Green amaranth) is also an annual herb having light green stem with height $60-80 \mathrm{~cm}$. A. caudatus is a flowering plant (Height: 3-8 ft) and can grow everywhere. A. hypochondriacus is an ornamental plant endemic to Mexico and used as a source of food. A. tricolor (Family: Amaranthaceae) is an annual flowering plant (Height: 2-3 ft) having rich purple flowers native to Bangladesh and used as a leafy vegetables.

Phytoconstituents: A total six species under the genus Amaranthus have been studied in this review and sixty five (1-65) molecules were reported as phytoconstituents (Figures 1-10). The reviewed medicinal plants are Amaranthus retroflexus, A. spinosus, A. viridis, A. caudatus, A. hypocondriacus, and A. tricolor.

Sesquiterpenes: Four sesquiterpenes having antioxidant properties were isolated from the medicinal plant A. retroflexus for example Amarantholidols A (1), Amarantholidols B (2), Amarantholidols C (3) and Amarantholidols D (4) (Figure 1) (Pacificao et al. 2008).

Glucosides: A bunch of sesquiterpenes glucosides such as Amarantholidoside I (5), Amarantholidoside II (6), Amarantholidoside II (7), Amarantholidoside IV (8), Amarantholidoside V (9), Amarantholidoside VI (10), Amarantholidoside VII (11) have been reported from the A. retroflexus (Figure 2) (Fiorentino et al., 2006). 
Prenylpropanoids: A few prenylpropanoids were also isolated with chemical structures solved from the species A. retroflexus under this genus like Ferulic acid (12), Umbelliferone (13), Apigenin (14),
Boropinic acid (15), 4-Geranyloxyferulic acid (16), 7-Isopentenyloxycoumarin (17), Auraptene (18), and Umbelliprenin (19) (Figure 3) (Fiorito et al., 2017; Touati et al., 2009; Okuyama et al., 2016).<smiles>C=CC(C)(O)C[C@H](O)/C=C(\C)CCC(O)C(C)(C)O</smiles><smiles>C=CC(C)(O)CC=CC(C)(O)CCC(O)C(C)(C)O</smiles><smiles>C=CC(C)(O)CC(O)C=C(C)CC=CC(C)(C)O</smiles><smiles>C=CC(C)(O)C[C@H](O)/C=C(\C)CCC(O)C(=C)C</smiles>

Figure 1. Sesquiterpenes from A. retroflexus.<smiles>C=C[C@](C)(O)C[C@@H](/C=C(\C)CC[C@H](O)C(C)(C)O)OC</smiles><smiles>C=C[C@](C)(O)CC(/C=C(\C)CC[C@H](O)C(=C)C)OCC</smiles><smiles>C=C[C@](C)(O)C[C@@H](/C=C(\C)CCC=C(C)C)OC</smiles><smiles>C=C[C@](C)(O)CC/C=C(\C)CC[C@@H](O[C@@H]1O[C@H](CO)[C@@H](O)[C@H](O)[C@H]1O)C(C)(C)O</smiles><smiles>C=C[C@](C)(O)CC/C=C(\C)CC[C@@H](O)C(C)(C)O[C@@H]1OC(CO)[C@@H](O)[C@H](O)[C@H]1O</smiles>

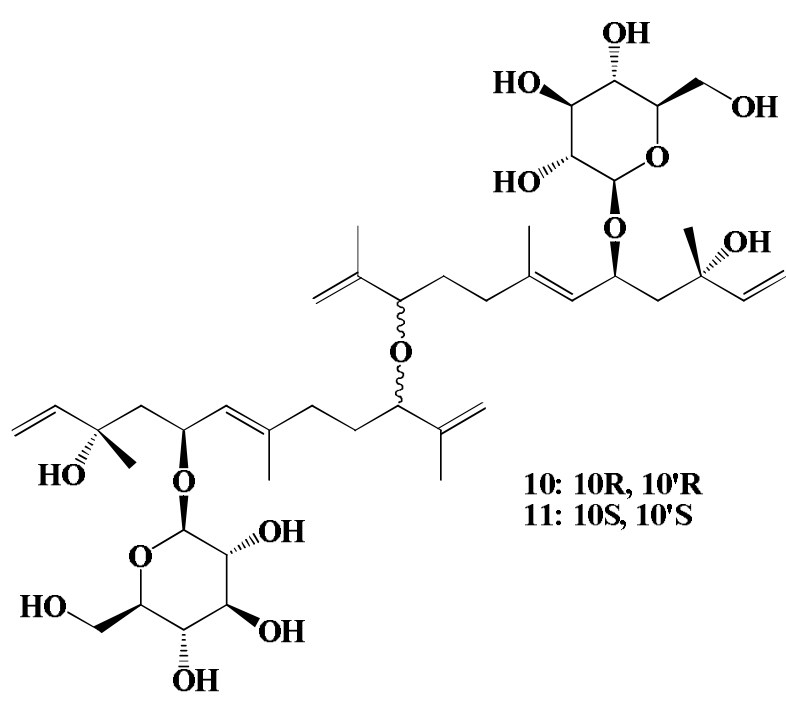

Figure 2. Glucosides from A.retroflexus. 


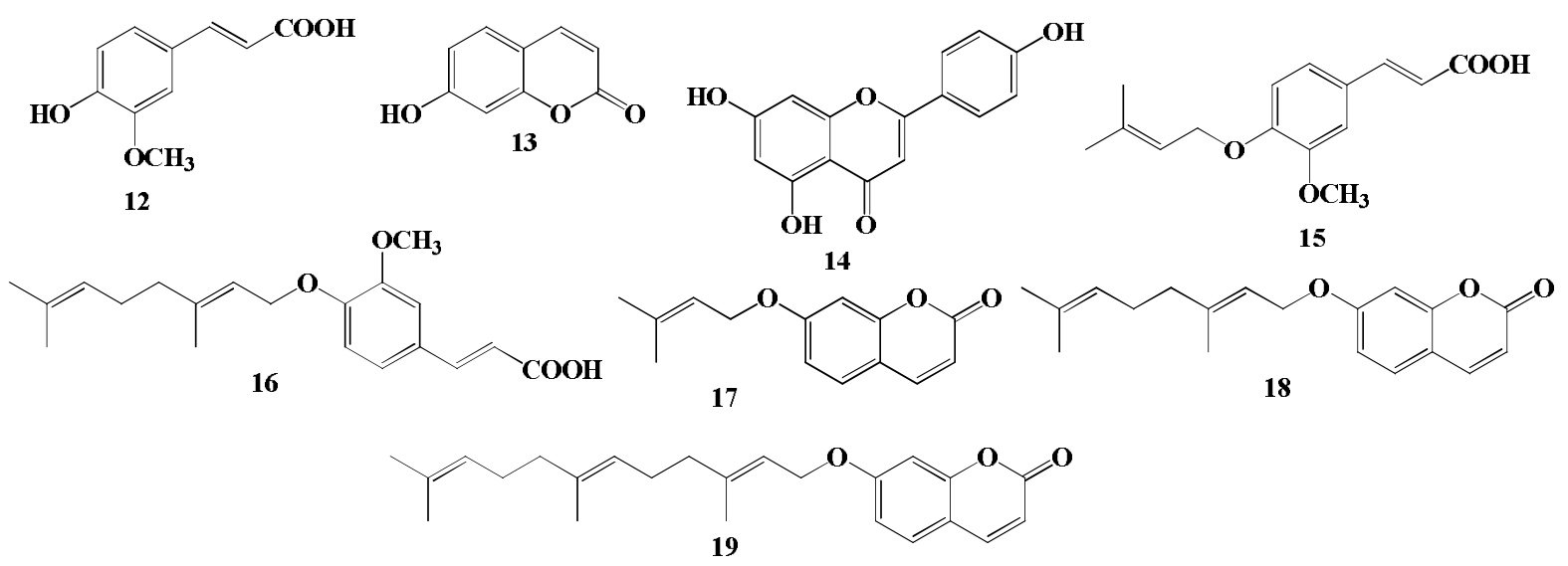

Figure 3. Prenylpropanoids from A. retroflexus.

Phenolic compounds: Two phenolic compounds such as Amaranthine (20) and Isoamaranthine (21) bearing anti-malarial activities are known from the medicinal plant A. spinosus (Figure 4) (Hilou et al. 2006; Stintzing et al. 2004).

Steroidal molecules: A group of steroidal molecules consist of anti-microbial functions have been found from A. viridis for example, Triacontanol (22), Palmitic acid (23), Spinasterol (24), Cycloeucalenol (25), Cholesterol (26), Oleanolic acid (27), 3,4-seco-olean-12-en-4-ol-3,28-dicarboxylic acid (28), Squalene (29), Trilonolein (30), Polyprenol (31), and Phytol (32) (Figure 5) (Hue et al., 2017, Ragasa et al., 2015; Naeem et al., 2012; Filho et al., 2007; Hoa et al., 2014; Xia et al., 2011; Rao et al., 1998; Basyuni et al., 2017; Santos et al., 2013).

Triterpnoids saponins and glycosides: A lot of triterpnoid saponins and glycosides for example 3-O$\alpha$-L-Arabinopyrano-syl-( $1 \rightarrow 2)$-[ $\beta$-D-glucopyranosyl$1 \rightarrow 6]-\beta-\mathrm{D}-2 \beta, 3 \beta, 6 \beta, 23$-tetrahydroxy-olean-12-en-28oic acid-28-O- $\beta$-D-glucopyranoside (33), 3-O- $\beta$-DArabinopyranosyl-( $1 \rightarrow 6)-\beta$-D-glucopyranosyl-

$2 \beta, 3 \beta, 6 \beta, 23$-tetrahy-droxyolean-12-en-28-oic acid$28-O$ - $\beta$-D-glucopyranoside $\quad$ (34), 3-O- $\beta$-DGlucopyranosyl-2 $\beta, 3 \beta$-dihydroxy-30-noroleane12,20(29)-diene-23,28-dioic acid 28-O- $\beta$-Dglucopyranosyl ester (35), 2 $\beta, 3 \beta$-Dihydroxy-30noroleane-12,20(29)-diene-23,28-dioic acid 28-O- $\beta$ D-glucopyranosyl ester (36), Oleanolic acid 3-O- $\beta$ D-glucopyranosyl-28-O- $\beta$-D-glucopyranoside (37), $2 \beta$-Hydroxyoleanolic acid 3-O- $\beta$-D-glucopyranosyl-
28-O- $\beta$-D-glucopyranoside (38),3- $O-\beta-\mathrm{D}-$ glucopyranosyl-2 $\beta, 3 \beta$-dihydroxyolean-12-ene-23,28dioic acid 28-O- $\beta$-D-glucopyranosyl ester (39), 3,4,5Trihydroxydehydro- $\alpha$-ionol-9- $O-\alpha-\mathrm{L}-$ rhamnopyranosyl-( $1 \rightarrow 6)-\beta-\mathrm{D}$-glucopyanoside

(40), 3,4,5-Trihydroxydehydro- $\alpha$-ionol-9- $O$ - $\beta$-D-

glucopyanoside (41), and 3-Oxo-4-hydroxy- $\beta$-ionyl9-O- $\beta$-D-glucopyranoside (42) are known to occur in A. caudatus (Figure 6) (Rastrelli et al. 1998).

Saponins: A few phytochemicals such as saponins were separated from A. hypochondriacus such as $\quad 3-O-\alpha$-L-Rhamnopyranosyl- $(1 \rightarrow 3)-\beta$-Dglucuronopyranosyl-2 $\beta, 3 \beta$-dihydroxyolean-12-ene28-oic acid 28-O- $\beta$-D-glucopyranosyl ester (43), 3$O-\alpha$-L-Rhamnopyranosyl-( $1 \rightarrow 3)-\beta$-Dglucuronopyranosyl-2 $\beta, 3 \beta$-dihydroxyolean-12-ene23-al-28-oic acid 28-O- $\beta$-D-glucopyranosyl ester (44), $\quad 3$ - $O$ - $\alpha$-L-Rhamnopyranosyl- $(1 \rightarrow 3)-\beta$-Dglucuronopyranosyl-2 $\beta, 3 \beta$-dihydroxy-30-norolean12,20(29)-dien-28-oic-acid 28-O- $\beta$-D-glucopyranosyl ester (45), 3- $O$ - $\alpha$-L-Rhamnopyranosyl- $(1 \rightarrow 3)-\beta$-Dglucuronopyranosyl-2 $\beta, 3 \beta$-dihydroxy-30-norolean12, 20(29)-dien-23-al-28-oic acid 28-O- $\beta$-Dglucopyranosyl ester (46), $2 \beta$-hydroxyoleanolic acid (47), Aterogenic acid (48), Prosapogenin (49), Thladioside H1 (50), Mixture of methyl-glucoside and prosapogenin (51) and Ciwujianoside $\mathrm{Cl}$ (52) (Figure 7) (Kohda et al., 1991). 


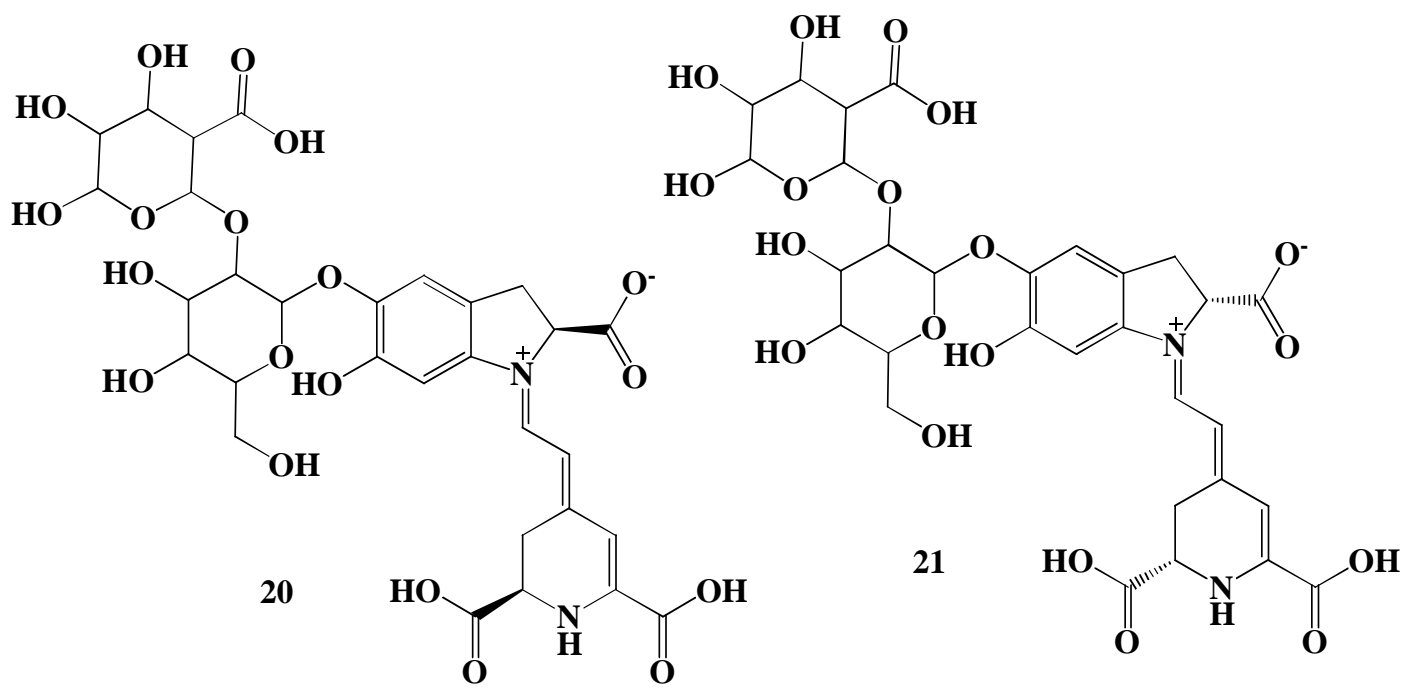

Figure 4. Phenolic compounds from A. spinosus.

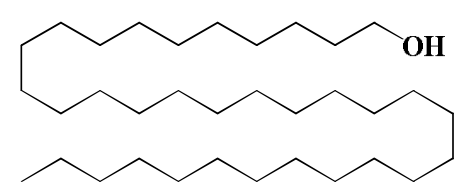

22<smiles>CCC(=O)O</smiles>

23<smiles>CCC(/C=C/C(C)[C@H]1CCC2C3=CCC4CC(O)CC[C@]4(C)C3CC[C@]21C)C(C)C</smiles><smiles>CC1C(C)C(C)([C@H]2CC[C@@]3(C(=O)O)CCC(C)(C)CC23C)CCC2C(C)(C)C(O)CCC12C</smiles>

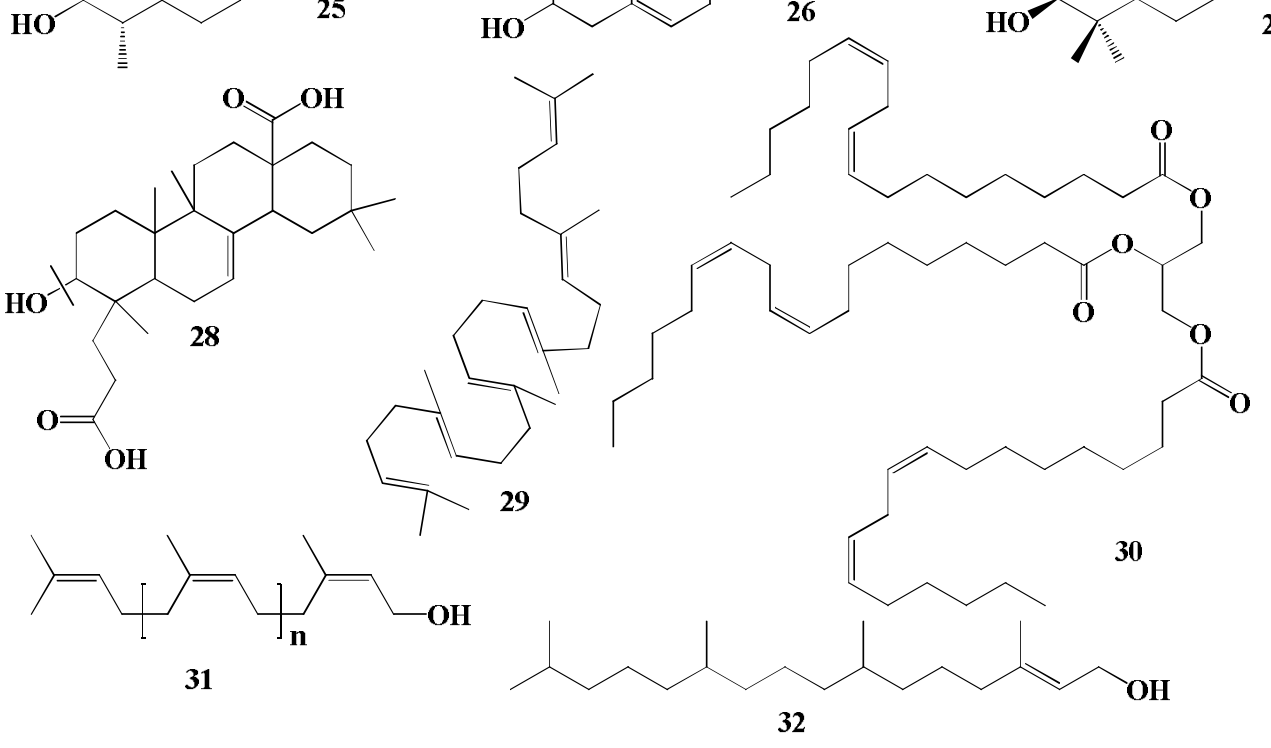

Figure 5. Steroidal compounds from A. viridis. 


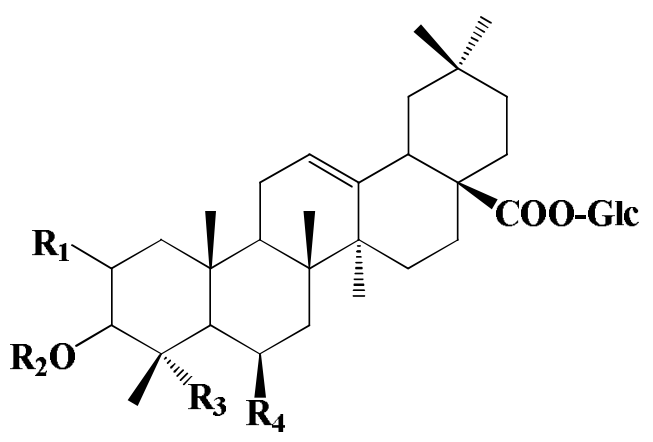

33: $\mathrm{R}_{\mathbf{1}}=\mathrm{OH}, \mathrm{R}_{\mathbf{2}}=$ GIc-Ara-GIc, $\mathrm{R}_{\mathbf{3}}=\mathrm{CH}_{2} \mathrm{OH}, \mathrm{R}_{\mathbf{4}}=\mathrm{OH}$

34: $\mathrm{R}_{1}=\mathrm{OH}, \mathrm{R}_{2}=$ Glc-Glc, $\mathrm{R}_{3}=\mathrm{CH}_{2} \mathrm{OH}, \mathrm{R}_{4}=\mathrm{OH}$

37: $\mathbf{R}_{\mathbf{1}}=\mathbf{H}, \mathbf{R}_{\mathbf{2}}=\mathbf{G l c}, \mathbf{R}_{\mathbf{3}}=\mathrm{CH}_{\mathbf{3}}, \mathbf{R}_{\mathbf{4}}=\mathrm{H}$

38: $\mathbf{R}_{1}=\mathrm{OH}, \mathrm{R}_{2}=\mathrm{Glc}, \mathrm{R}_{\mathbf{3}}=\mathrm{CH}_{3}, \mathrm{R}_{\mathbf{4}}=\mathrm{H}$

39: $R_{1}=O H, R_{2}=$ Glc-Glc, $R_{3}=C O O H, R_{4}=H$

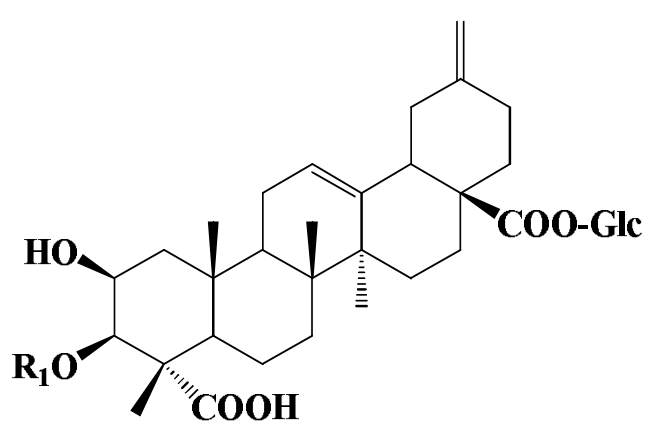

36: $R_{1}=H$<smiles>[R]O[C@H](C)/C=C/[C@]1(O)C(C)(C)C[C@H](O)CC1(O)O</smiles>

40: $R$ = Glc-Rha 41: $R=$ Glc

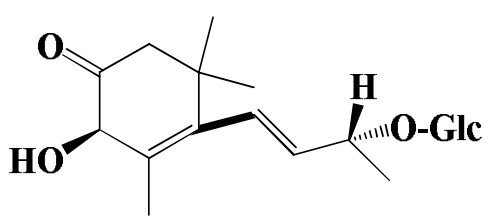

42

Figure 6. Triterpnoids saponins and glycosides from A. caudatus.

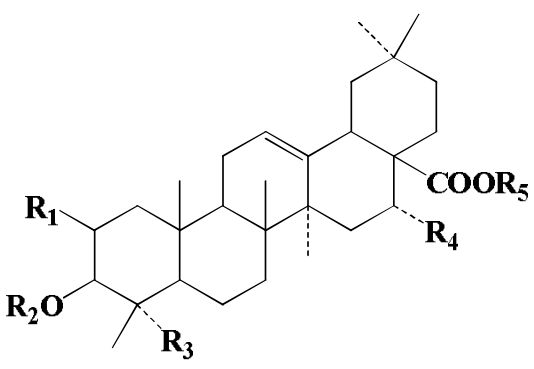

43: $\mathbf{R}_{\mathbf{1}}=\mathbf{O H}, \mathbf{R}_{\mathbf{2}}=\mathbf{G l c U A - R h a ,} \mathbf{R}_{\mathbf{3}}=\mathbf{C H}_{\mathbf{3}}, \mathbf{R}_{\mathbf{4}}=\mathbf{H}, \mathbf{R}_{\mathbf{5}}=\mathbf{G l c}$ 44: $\mathbf{R}_{\mathbf{1}}=\mathrm{OH}, \mathbf{R}_{\mathbf{2}}=$ GlcUA-Rha, $\mathbf{R}_{\mathbf{3}}=\mathbf{C H O}, \mathbf{R}_{\mathbf{4}}=\mathbf{H}, \mathbf{R}_{\mathbf{5}}=\mathbf{G l c}$ 47: $\mathbf{R}_{\mathbf{1}}=\mathrm{OH}, \mathbf{R}_{\mathbf{2}}=\mathbf{H}, \mathbf{R}_{\mathbf{3}}=\mathrm{CH}_{3}, \mathbf{R}_{\mathbf{4}}=\mathbf{H}, \mathbf{R}_{5}=\mathbf{H}$ 48: $\mathrm{R}_{1}=\mathrm{OH}, \mathrm{R}_{\mathbf{2}}=\mathrm{H}, \mathrm{R}_{\mathbf{3}}=\mathrm{CH}_{3}, \mathrm{R}_{\mathbf{4}}=\mathrm{OH}, \mathrm{R}_{\mathbf{5}}=\mathrm{CH}_{3}$ 49: $\mathbf{R}_{1}=\mathbf{O H}, \mathbf{R}_{\mathbf{2}}=$ GlcUA-Rha, $\mathbf{R}_{3}=\mathrm{CH}_{3}, \mathbf{R}_{\mathbf{4}}=\mathbf{H}, \mathbf{R}_{5}=\mathbf{H}$ 50: $\mathbf{R}_{\mathbf{1}}=\mathbf{H}, \mathbf{R}_{\mathbf{2}}=$ GlcUA-Glc, $\mathbf{R}_{\mathbf{3}}=\mathrm{CHO}, \mathbf{R}_{\mathbf{4}}=\mathbf{H}, \mathbf{R}_{\mathbf{5}}=$ Xyl-Rha-Xyl-Xyl 51: $\mathbf{R}_{1}=\mathbf{O H}, \mathbf{R}_{\mathbf{2}}=$ GlcUA-Rha, $\mathbf{R}_{\mathbf{3}}=\mathrm{CHO}, \mathbf{R}_{\mathbf{4}}=\mathbf{H}, \mathbf{R}_{\mathbf{5}}=\mathbf{H}$<smiles>[R10]OC(=O)C12CCC(=C)CC1C1=CCC3C(C)(CCC4C([R3])(C)C([R20])C([R10])CC43C)C1CC2</smiles>

45: $\mathbf{R}_{\mathbf{1}}=\mathrm{OH}, \mathrm{R}_{\mathbf{2}}=$ GlcUA-Rha, $\mathrm{R}_{\mathbf{3}}=\mathrm{CH}_{\mathbf{3}}, \mathbf{R}_{\mathbf{4}}=\mathbf{G l c}$ 46: $R_{1}=O H, R_{2}=$ GlcUA-Rha, $R_{3}=$ CHO, $R_{4}=$ Glc 52: $\mathbf{R}_{1}=\mathbf{H}, \mathbf{R}_{\mathbf{2}}=$ Ara, $\mathbf{R}_{3}=\mathrm{CH}_{3}, \mathbf{R}_{\mathbf{4}}=$ Glc-Glc-Rha

GlcUA: Beta-D-Glucuronopyranosyl Rha: Alpha-L-Rhamnopyranosyl Glc: Beta-D-Glucopyranosyl Ara: Alpha-L-Arabinopyranosyl Xyl: Beta-D-Xylopyranosyl

Figure 7. Saponins from A. hypochondriacus.

Fatty acids: Four fatty acids for example Linolenic acid (53), Lignoceric acid (54), Arachidic acid (55), and 24-Methylenecycloartenol (56) with were also isolated from, A. tricolor under this genus (Figure 8) (Fernando et al., 1984; Otto et al. 2014).
Flavonoids: Four flavonoidal phytoconstituents like Gallic acid (57), Caffeic acid (58), Rutin (59), and Quercetin (60) were also isolated from $A$. caudatus (Figure 9) (Paranthaman et al., 2012; Reckziegel et al., 2016; Zhao et al., 2014). 
Miscellaneous compounds: A few other compounds have also been isolated from A. spinosus such as Caffeoylquinic acid (61), Coumaroylquinic rutinoside (64), and Quercetin 3-O-glucoside (65) (Figure 10) (Stintzing et al., 2004; Iwashina et al., 2012).

acid (62), Feruloylquinic acid (63), Quercetin 3-O-
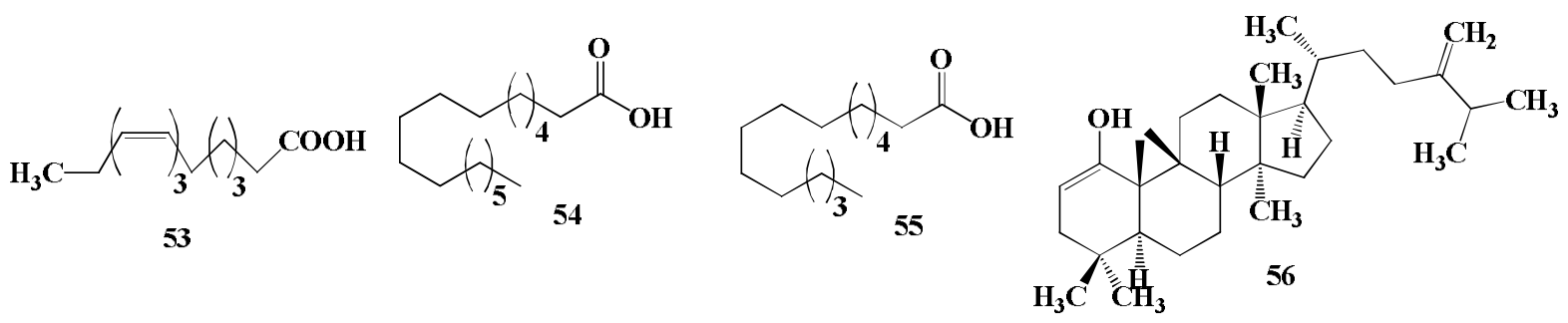

Figure 8. Fatty acids from A. tricolor.<smiles>O=C(O)c1cc(O)c(O)c(O)c1</smiles>

57<smiles>O=C(O)/C=C/c1ccc(O)c(O)c1</smiles>

58<smiles>O=c1c(O)c(-c2ccc(O)c(O)c2)oc2cc(O)cc(O)c12</smiles>

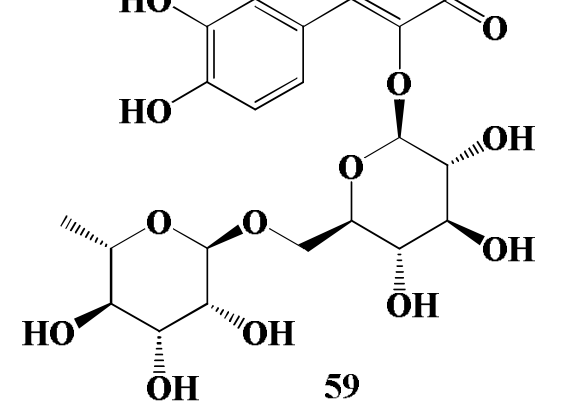

Figure 9. Flavonoids from A. caudatus.<smiles>O=C(/C=C/c1ccc(O)c(O)c1)OC1(C(=O)O)C[C@H](O)[C@H](O)C[C@H]1O</smiles><smiles>[2H][C@@]1(C(=O)O)C[C@@H](O)C(OC(=O)/C=C/c2ccc(O)cc2)C[C@@H]1O</smiles><smiles>COc1cc(/C=C/C(=O)OC2C[C@](O)(C(=O)O)CC(O)C2O)ccc1O</smiles><smiles>CC1OC(OCC2OC(Oc3c(-c4ccc(O)c(O)c4)oc4cc(O)cc(O)c4c3=O)C(O)C(O)C2O)C(O)C(O)C1O</smiles>

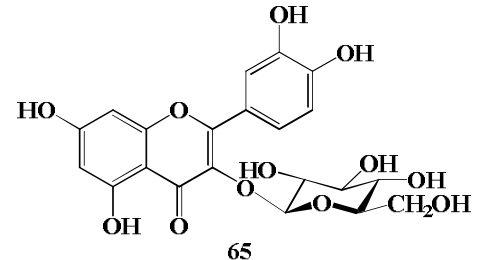

Figure 10. Miscellaneous compounds from A. spinosus. 


\section{Conclusion}

Phytoconstituents from six species under the genus Amaranthus have been studied. Different molecules with structurally distinctive characteristics have been reported from these medicinal plants.

\section{References}

Hussain, M.M. 2018. A short review on phytoconstituents from the genera Albizzia and Erythrina. Bangladesh Pharm. J. 21, 160-172.

Hussain, M.M., Tuhin, M.T.H., Akter, F. and Rashid, M.A. 2016a. Constituents of Erythrina-a potential source of secondary metabolites: A review. Bangladesh Pharm. J., 19, 237-253.

Hussain, M.M., Tahia, F. and Rashid, M.A. $2016 \mathrm{~b}$. Secondary metabolites from some species of Albizzia: A review. Bangladesh Pharm. J. 19, 1-8.

Hussain, M.M., Dastagir, M.G., Billah, A.H.M.M. and Ismail, M. 2011. Alpinum isoflavone from Erythrina stricta Roxb. Bol. Latinoam. Caribe Plant. Med. Aromat. 10, 88-90.

Hussain, M.M., Mughul, M.M.R., Alam, M.M., Dastagir, M.G., Billah, A.H.M.M. and Ismail, M. 2010. Antimicrobial activity of $n$-hexane and ethyl acetate extracts of Erythrina stricta Roxb. Bangladesh $J$. Microbiol. 27, 65-66.

Hussain, M.M., Rahman, M.S., Jabber, A. and Rashid, M.A. 2008. Phytochemical and biological investigation of Albizzia lebbek Benth. Bol. Latinoam. Caribe Plant. Med. Aromat. 7, 273-278.

Billah, A.H.M.M., Hussain, M.M., Dastagir, M.G., Ismail, M. and A. Quader, A. 2013. $\alpha$-Spinasterol from Amaranthus spinosus stem. Bol. Latinoam. Caribe Plant. Med. Aromat. 12, 15-17.

Ismail, M., Hussain, M.M., Dastagir, M.M., Billah, M. and Quader, A. 2010. Phytochemical and antimicrobial investigation of Luffa cylindrical. Bol. Latinoam. Caribe Plant. Med. Aromat. 9, 327-332.

Pacifico, S., Abrosca, B.D., Golino, A., Mastellone, C., Piccolella, S., Fiorentino, A., P. Monaco, P. 2008. Antioxidant evaluation of polyhydroxylated nerolidols from redroot pigweed (Amaranthus retroflexus) leaves. LWT-Food Sci. Technol. 41, 1665-1671.

Fiorentino, A., Greca, M. D., Abrosa, B.D., Golino, A., Pacifico, S., Izzo, A. and Monaco, P. 2006. Unusual sesquiterpene glucosides from Amaranthus retroflexus. Tetrahedron 62, 8952-8958.
Fiorito, S., Epifano, F., Palmisano, R., Genovese, S. and Taddeo, V.A. 2017. A re-investigation of the phytochemical composition of the edible herb Amaranthus retroflexus L. J. Pharm. Biomed. Anal. 143, 183-187.

Touati, E., V. Michel, V., Correia, M., Menghini, L., Genovese, S., Curini, M. and Epifano, F. 2009. Boropinic acid, a novel inhibitor of Helicobacter pylori stomach colonization. J. Antimicrobial Chemother. 64, 210-211.

Okuyama, S., Semba, T., Toyoda, N., Epifano, F., Genovese, S., Fiorito, S., Taddeo, V.A., Sawamoto, A., Nakajima, M. and Furukawa, Y. 2016. Auraptene and other prenyloxyphenylpropanoids suppress microglial activation and dopaminergic neuronal cell death in a lipopolysaccharide-induced model of Parkinson's disease. Int. J. Mol. Sci. 17, 1716.

Hilou, A., Nacoulma, O.G. and Guiguemde, T.R. 2006. In vivo anti-malarial activities of extracts from Amaranthus spinosus L. and Boerhaavia erecta $L$. in mice. J. Ethnopharmcol. 103, 236-240.

Stintzing, F.C., Kammerer, D., Schieber, A., Adama, H., Nacoulma, O.G. and Carle, R. 2004. Betacyanins and phenolic compounds from Amaranthus spinosus L. and Boerhaavia erecta L. Z. Naturforsch. 59c, 1-8.

Hue, C.T., Oanh, N.T.T., Giap, T.H., Hang, N.T.M., Mishchenko, N.P., Fedoreev, S.A. Spiridovich, E.V., Minh, C.V., Voung, N.Q. and Thanh, L.N. 2017. Metabolites of the Vietnamese plant Amaranthus viridis. Chem. Nat. Compd. 53, 1150-1151.

Ragasa, C.Y., Austria, J.P.M., Subosa, A.F., Torres, O.B. and Shen, C.C. 2015. Chemical constituents of Amaranthus viridis. Chem. Nat. Compd. 51, 146-147.

Naeem, M. and Khan, M.M.A., Moinuddin. 2012. Triacontanol: a potent plant growth regulator in agriculture. J. Plant Interactions, 7, 129-142.

Filho, F.A.S., Lima, M.A.S., Bezerra, A.M.E., Filho, R.B., Silveiro, E.R. 2007. A labdane diterpene from the aerial parts of Egletes viscosa Less. J. Braz. Chem. Soc. 7, 1374-1378.

Hoa, N.T., Dien, P.H., Quang, D.N. 2014. Cytotoxic steroids from the stem barks of Pandanus tectorius. Res. J. Phytochem. 8, 52-55.

Xia, E.Q., Wang, B.W., Xu, X.R., Zhu, L., Song, Y. and Li, H.B. 2011. Microwave-assisted extraction of oleanolic acid and ursolic acid from Ligustrum lucidum Ait. Int. J. Mol. Sci. 12, 5319-5329. 
Rao, C.V., Newmark, H.L. and Reddy, B.S. 1998. Chemopreventive effect of squalene on colon cancer. Carcinogenesis 19, 287-290.

Basyuni, M., Sagami, H., Baba, S., Putri, L.A.P., Wati, R. and $\mathrm{Oku}, \mathrm{H}$. 2017. Salinity alters the poly isoprenoid alcohol content and composition of both salt-screening and non-salt screening mangrove seedlings. HAYATI J. Biosci. 24, 206-214.

Santos, C.C.D.M.P., Salvadori, M.S., Mota, V.G., Costa, L.M., Almeida, A.A.C.D., Oliveira, G.A.L.D., Costa, J.P., Sousa, D.P.D., Freitas, R.M.D. and Almeida, R.N.D. 2013. Antinociceptive and antioxidant activities of phytol in vivo and in vitro models. Neurosci. J. 949452.

Rastrelli, L., Aquino, R., Abdo, S., Proto, M., Simone, F.D. and Tommasi, N.D. 1998. Studies on the constituents of Amaranthus caudatus Leaves: Isolation and structure elucidation of new triterpenoid saponins and ionol-derived glycosides. J. Agric. Food. Chem. 46, 1797-1804.

Kohda, H., Tanaka, S., Yamaoka, Y. and Ohhara, Y. 1991. Saponins from Amaranthus hypochondriacus. Chem. Pharm. Bull. 39, 2609-2612.

Fernando, T. and Bean, G. 1984. Fatty acids and sterols of Amaranthus tricolor L. Food Chem. 15, 233-237.
Otto, J.R., Freeman, M.J., Aduli, B.S.M., Nichols, P.D., Lane, P.A. and Aduli, A.E.O.M. 2014. Reproduction and fertility parameters of dairy cows supplemented with omega-3 fatty acid rich canola oil. Annual Res. Rev. Biology 4, 1611-1636.

Paranthaman, R., Kumar, P.P. and Kumaravel, S. 2012. GC-MS analysis of phytochemicals and simultaneous determination of flavonoids in Amaranthus caudatus by RP-HPLC. J. Anal. Bioanal. Tech. 3, 147.

Reckziegel, P., Dias, V.T., Benvegnu, D.M., Boufleur, N., Barcelos, R.C.S., Segat, H.J., Pase, C.S., Santos, C.M.M.D., Flores, E.M.M. and Burger, M.E. 2016. Antioxidant protection of gallic acid against toxicity induced by $\mathrm{Pb}$ in blood, liver, and kidney of rats. Toxicol. Rep. 3, 351-356.

Zhao, C., Lu, Z., Li, C., He, X., Li, Z., Shi, K., Yang, L., Fu, Y. and Zu, Y. 2014. Optimization of ionic liquid based simultaneous ultrasonic- and microwaveassisted extraction of rutin and quercetin from leaves of velvet leaf (Abutilon theophrasti) by response surface methodology. Scientific World J. 283024.

Iwashina, T., Smirnov, S.V., Damdinsuren, O. and Kondo, K. 2012. Flavonoids from Raeumuria soongarica (Tamariaceae) in Mongolia. Bull. Natl. Mus.Nat. Sci., Ser. B 38, 189-195. 